\title{
The Implementation and Validation of a Virtual Environment for Training Powered Wheelchair Manoeuvres
}

\author{
Nigel W. John, Serban R. Pop, Thomas W. Day, \\ Panagiotis D. Ritsos, Member, IEEE, and Christopher J. Headleand
}

\begin{abstract}
Navigating a powered wheelchair and avoiding collisions is often a daunting task for new wheelchair users. It takes time and practice to gain the coordination needed to become a competent driver and this can be even more of a challenge for someone with a disability. We present a cost-effective virtual reality (VR) application that takes advantage of consumer level VR hardware. The system can be easily deployed in an assessment centre or for home use, and does not depend on a specialized high-end virtual environment such as a Powerwall or CAVE. This paper reviews previous work that has used virtual environments technology for training tasks, particularly wheelchair simulation. We then describe the implementation of our own system and the first validation study carried out using thirty three able bodied volunteers. The study results indicate that at a significance level of $5 \%$ then there is an improvement in driving skills from the use of our VR system. We thus have the potential to develop the competency of a wheelchair user whilst avoiding the risks inherent to training in the real world. However, the occurrence of cybersickness is a particular problem in this application that will need to be addressed.
\end{abstract}

Index Terms—Virtual Reality, Virtual Environment, Simulation, Wheelchair Navigation

\section{INTRODUCTION}

A CCORDING to the census of England and Wales carried out in 2011,1.9\% of the population use a wheelchair, an estimated 1.2 million people. Other countries will have proportionally similar numbers within their population. Demand for wheelchairs, particularly powered wheelchairs, is growing, with the powered wheelchair market in the United States alone expected to grow to $\$ 3.9$ billion by 2018 .

When an individual is referred to their local Wheelchair Centre they will be assessed by an occupational therapist for their competency to safely use a powered wheelchair. Learning the necessary driving skills can be a daunting task, particularly for individuals with severe, or multiple motor limitations [13], [35]. Significant challenges in this training are evident, such as the need to improve a persons spatial awareness, reaction times, familiarisation with and general confidence in the wheelchairs operation. Although modern buildings increasingly cater for people with disabilities [39], there are still many areas where wheelchair access cannot be solved architecturally and rely on learning to control the device. However, there is no common standard for assessment and training of a person for the use of a powered chair and most Wheelchair Services have developed their own protocols, typically involving a driving assessment in both interior and exterior surroundings.

There has been some previous research into using virtual reality (VR) to help train wheelchair users, but price and

- N.W. John, S.R. Pop and T.W. Day are with the Department of Computer Science, University of Chester, UK e-mail: (see http://medicalgraphics.org/).

- P.D. Ritsos is with Bangor University, UK.

- C.J. Headleand is with University of Lincoln, UK.

Manuscript received February, 2017. technology limitations have been a barrier to commercial adoption (see details in section 2). However, affordable, high fidelity interfaces for VR such as the Oculus Rift head mounted display are now becoming readily available. With these new technological advances it is timely to investigate a more intuitive, immersive virtual environment for training users of powered wheelchairs that can easily be deployed. We hypothesise that a serious game that utilises affordable modern interface technologies can provide a safe environment in which a new user of a powered wheelchair can quickly learn how to operate it, and navigate it, in a variety of different scenarios. The particular contribution of this paper is to detail the design and implementation of a VR training environment that exploits the new generation of HMDs. Although aimed at a specific user domain lessons learned will be relevant to other training applications. Further we present one of the first validation studies that suggest that these devices can be successfully deployed for accelerating task training.

The structure of the paper is as follows: Section 2 discusses related work on training in VR, and in particular VR wheelchair simulators. The main ongoing challenges are identified. Section 3 describes Wheelchair-VR, the system that we have developed to explore the above hypothesis. Section 4 presents the first validation study on the use of Wheelchair-VR with able-bodied volunteers and Section 5 discusses its results. Finally the paper ends with our conclusions and a discussion of future work.

\section{Related Work}

Our approach in examining related work is twofold. Firstly, we briefly highlight the importance of VR as a training tool, 
looking into various usage scenarios. Secondly, we examine in detail prior work on Wheelchair simulators.

\subsection{Training in VR}

VR has been utilised in various training scenarios, spanning from education [3], manufacturing [33], maintenance [27], business [25], and sports [4], to post-disaster triage [2], safety training for firefighters [5] and the mining industry [41], [42]. A recent survey by Slater and Sanchez-Vives [37] looks at the current evidence for the utility of VR across an extensive range of application areas. They state that new affordable systems will facilitate not only the reach to final consumers but also to more developers and research groups, resulting in a much wider range of applications and generation of content for VR that will emerge in the near future. Of particular interest to our work, are prior endeavours that focus on two aspects: a) skills transfer and b) motor skills training through VR.

Regarding skills transfer, early investigations, such as the one from Kozak et al. [26] who examined pick-and-place maintenance tasks using early VR interfaces, indicated that skills transfer from VR to the real world is limited. Likewise, motor skills training was deemed insufficient. The authors', believed that the state-of-the-art of the VR interfaces, at that time, was the main obstacle. On the contrary, later efforts, such as the ones from Loftin and Kenney [29], Wilson et al. [45] and Seymour et al. [36] demonstrated positive results, regarding skills transfer. The recent launch of many cost effective VR devices is likely to support a continuation of this trend, which is supported by the results that we describe in this paper.

Many researchers have investigated the potential of VR for training and transfer of motor skills. For example within the domain of rehabilitation, Adamovich et al. [1] review the use of VR in sensorimotor training in neurorehabilitation up to 2009. They argue that VR may be an optimal tool for designing rehabilitation environments that are hard to recreate in the physical world cater and can be used for mass practice. They add that VR can provide automatic modulation of task difficulty in motor-skills tasks, and high levels of engagement and motivation. Furthermore, Brütch et al. [7] discuss how VR offers powerful therapy options in paediatric rehabilitation, and that the VR scenario they explore has an a learning effect similar to verbal instructions from therapists. De Bruin et al. [11] explore the use of VR training for motor control through dancing for older adults, highlighting that VR offers a safe environment for training, the opportunity for independent exploration and consistency in delivering therapeutic protocols. Finally, Molina et al. [32] provide a comprehensive review on the use of VRbased gaming for rehabilitating physical exercises, for older adults. They also highlight the need for further investigation on the potential benefits of VR training, compared to more traditional methods. There are many more published examples.

There are also many examples of where VR has been used to train the motor skills required to carry out specific medical and surgical procedures (e.g., [6], [10]), including our own work with our collaborators [23], [43] and [30]. Formal validation studies carried out within these projects demonstrated that there is skill transfer from VR to the real world scenario, and that it is possible to discriminate between users with different levels of skill.

\subsection{Wheelchair Simulators}

An extensive review of wheelchair simulators was made in 2009 by Pithon et al. [34]. The studies reviewed at that time already provided some evidence that VR training can be effective and increase the motivation of the users. However, it was evident that the models of wheelchair behaviour needed to be improved as the visual interface and the integration of feedback was often not explained. Pithon presented evidence that improvements were needed to the mechanical systems being used, the physics of the wheelchair motion, and also more use of sound feedback. In fact, the first attempts to use VR to help train users of powered wheelchairs took place in the late 1990s, but were hampered by the limitations in available technology. Inman et al. [21] focused on developing entertaining environments that would motivate children. They demonstrated that certain aspects of the childrens driving skills did improve through training in VR. Interestingly, they noted that many of the children chose to look at a large monitor rather than using the head-mounted display (HMD). However, the resolution of HMDs at the time was far inferior to that of monitors. A non-immersive VR Training System for disabled children was also developed by Desbonnet et al. [12], who provided good face validity by using the actual wheelchair controller fitted to the chair. Yet the visual realism achieved and the modeled wheelchair behaviour were not sufficient due to the limitations in polygon count and the real time modelling techniques available in the software used to develop this early system. A mechanical rig was also noted as being needed to provide realistic feedback. These were highlighted as important areas to address in future work. Recent studies by Linden et al. [28] provide further evidence of the effectiveness and value of virtual wheelchair training simulators for children.

Harrison et al. [17] carried out a comprehensive study to explore the use of VR in the assessment and training of powered wheelchair users. However, the experience was again non-immersive, using a computer monitor to display the virtual environment with user control through a games joystick, or in some cases, the actual wheelchair joystick. They modeled a single large room that was populated with several chairs and tables. Able bodied, experienced, and inexperienced powered wheelchair users took part in experiments where they were required to complete various manoeuvres: drive the virtual wheelchair forward in a straight line for ten metres; reverse the wheelchair in a straight line for two metres; drive the wheelchair into and out of an enclosed space; complete a $180^{\circ}$ turn around a stationary object; completing a slalom; and stop the wheelchair suddenly to command (we have implemented similar tasks to these in our Wheelchair-VR system). Experienced users were given an opportunity to explore a real-life environment before using the VR system. Practice using the interface device (a wheelchair joystick in this scenario) also took place beforehand. The findings were that generally users were quicker in real life than in VR, the slowest in VR being the 
driving into a gap, 180 degree turn and slalom. The number of collisions and individual manoeuvres required in VR were considerably more than in reality and were attributed to slight differences to the real life chair. The easiest task was the manoeuvring in straight lines whereas the hardest was the slalom where the finest control was required. Individual interviews were also conducted after all tests were over. The authors conclude that whilst showing potential, the virtual environments need to be less challenging if they are to represent a motivating and effective means of improving performance.

Later work by Inman et al. [22] produced a simulator called WheelchairNet designed to help orthopedically challenged individuals learn to more safely and effectively operate a powered wheelchair by practicing wheelchair driving manoeuvres in three different virtual environments. The simulation is controlled mainly with arrow keys on the keyboard. The computer monitor view is first person with the ability to pan up and down to the point where the wheelchair users legs are visible. There are options for adjusting the wheelchair configuration before the simulation begins. The purpose in each environment is to navigate and avoid obstacles until a certain point in reached, a portal whereby the user is taken to the next level. A key press reveals a head up display with information such as current time, level, and a score. The software supports multiple users, which is a useful feature. In a study using children with physical disabilities, use of WheelchairNET over time was shown to improve the skills required in the actual world for independent mobility as well as practical skills such as crossing a street. The children were able to learn functional skills in the virtual world without the risks associated with real word training. WheelchairNet is freely available but no longer being developed.

Another important factor in increasing immersion can be to augment the visual response with haptics. The first use of a force feedback system in a wheelchair training environment is described by Harrison et al. [18], [19]. Using a sophisticated platform with embedded electromechanical components, they were able to use data from the virtual environment on the surface properties and gradient beneath the wheels to deliver controlled torque to the wheelchair rims and so enable the appropriate haptic feedback. The wheelchair platform was positioned in front of a curved screen to provide high resolution graphical output. The system is not aimed at training, but to give architects and designers a physical sensation on how a planned development incorporates disability access. Grant et al. [16] note that the assessment of accessibility requires more realistic models than mere training tools, and should effectively "communicate the experience of wheelchair operation", implying a requirement for a higher level of immersion.

Virtual environments can also be used to prototype new parts for a wheelchair. Cooper et al. [9] compared a new isometric joystick for a powered wheelchair with the traditional position sensing joystick in this manner. They found that performance in the virtual environment was representative of driving ability on the real environment. Mahajan et al. [31] explored VR simulations using large screens (powerwalls) for driving assessments.

Based on our review of the literature the need for three main areas of development have been identified:

1) Environments that actively train speed modulation and wheelchair control in complex spaces, particularly crowded areas.

2) Interfaces which allow for natural interaction and accessibility to physical interfaces such as switches, door handles, swipe card mechanisms, etc.

3) Force-feedback, allowing the user to feel the virtual environment through the wheelchair. While some forcefeedback has been shown to be effective in wheelchair simulation [17], it is an area which has received relativity little attention.

We have addressed the first two of these areas in our current implementation of Wheelchair-VR.

\section{Methods and Tools}

This section provides an overview of the design and implementation of Wheelchair-VR, including the wheelchair configuration options and the virtual environment tasks. We use the Unity 3D game development platform (Unity Technologies, San Francisco, CA), which will operate on any standard PC or Mac platform.

\subsection{Design Considerations}

Wheelchair-VR was designed and tested with subject matter experts from four different regional Wheelchair Centres. They provided initial face validation on the utility of the application and suggestions for further improvement. Training scenarios such as manoeuvring over and around access ramps, driving through doors into tight corridors; and driving down tight paths with curbs were suggested and subsequently implemented as a result of these sessions. The possibility of having more abstract tasks that could be used to practice hand eye coordination was also discussed, which led to some scenarios that would not be possible in the real world. For example, one room in the virtual world is filled with red and blue balls floating at different heights. The task is to collect all the blue balls by driving into them, but avoid all the red balls.

The experts also used the first prototype of WheelchairVR with a HMD to complete a navigation task allowing qualitative feedback to be collected. Overall, the simulation was characterized as accurate in its operation, with the feeling of immersion, interaction and environment quality as strong points. A series of parameters were identified for adjustment, namely the turning speed and braking speed of the virtual chair.

One of the Wheelchair Centres also informed us that they measure the reaction time of their clients. They found through experience that a reaction time of over one second was a strong indicator that the client would not be able to control a wheelchair with any proficiency. This information was used later to screen volunteers in the validation study.

\subsection{Building the Virtual Powered Wheelchair}

We have restricted our simulation to Class 2 wheelchairs, which can only be used on the pavement if outside and are limited to traveling at $4 \mathrm{mph}$. Figure 1 shows an example. Class 3 wheelchairs are allowed on the road at up to $8 \mathrm{mph}$ but tend to be larger and require extra safety features. 


\subsubsection{The Control Unit}

The control unit most commonly used on a powered wheelchair is a single joystick for all of the wheelchair's movement, with buttons for other functions. It can be fixed to the left or right side of the wheelchair, sometimes hinged so that it moves away. Proportional control can be used so that the amount of push or pull controls the speed. Controllers operated by the head, chin or tongue, or sip and puff style, are outside the scope of the current project. To replicate this control unit, a convenient off-the-shelf solution was to use a PC gamepad device such as the Microsoft XBox One controller. One of the thumbsticks on the XBox controller can then be used as the wheelchair joystick for selecting the direction of motion. To increase the face validity of this approach, a joystick grip from a real wheelchair controller was fitted on top of the thumbstick ${ }^{1}$ - as can be seen in Figure 4. The trainee uses Wheelchair-VR in a seated position with the XBox controller in a fixed position corresponding to where it would be were it fixed to the arm of a real wheelchair.

\subsubsection{The Drive System}

The drive system depends on which set of wheels are powered by the motor, and there are several configurations available:

- Mid wheel drive where the drive wheels sit in the middle of a set of six, making the chair more manouverable and minimising the turning circle. Six wheels help maintain stability on slopes but performs better on solid and even ground. Not usually used outdoors.

- Front wheel drive where the drive wheels are at the front of the wheelchair. This system is best for steering around tight corners and for use on uneven terrain (as the large front wheels can climb over bumps and obstacles). It is more stable on slopes but can also be more difficult to steer at high speed.

- Rear wheel drive where the drive wheels are at the rear of the wheelchair with free motion of pivoting front caster wheels. This is the most common system. The turning circle is larger than other systems, but many people find it easier to drive in a straight line. This system also helps maintain smoothness on rough terrain. Often used with anti-tippers to keep the wheelchair from tipping backwards, as the majority of the wheelchairs weight is at the back.

- Wheelchairs that can switch between front and rear wheel drive.

- Four wheel drive, which is the best for rough terrain and steps.

A design decision was made to move to a kinematic model using Unity's built-in character controller. The character controller produces physics-like interactions, without making use of rigid bodies. By taking this approach our system can be configured to behave according to any of the above configurations through the modification of a small number of logical parameters.

Slope Limit: varying the maximum incline that the chair can successfully navigate;

1. We have also designed a joystick grip that snaps onto the thumbstick and can be fabricated with a 3D printer.
Pivot Centre: changing where the chair pivots from during a turn, allowing the simulation of rear, and centre wheel drive chairs;

Acceleration: varying the acceleration curve from zero to maximum speed;

Turn Speed: altering the rotational speed while turning;

Stopping Speed: changing how quickly the chair stops when power is no longer being applied.

By altering these default parameters all commercially available chairs can be simulated within an acceptable margin of error. While the study described in this paper used a simulated rear-wheel drive chair (based on the Spectra XTR2 shown in Figure 1) other configurations have been successfully simulated.

In previous experiments a physics-based wheelchair simulator was used [20]. However, physics-based models are notoriously hard to configure, and settings needed to be extensively modified for each individual wheelchair configuration. This was to ensure that the weight distribution, friction curves, torque, and drag characteristics were correctly specified. If any of these settings were marginally incorrect, the chair would not move realistically.

As our collaborators at the Wheelchair Centres require a system that is easily configurable to a number of wheelchair models, this presents an unwanted limitation. Secondly, there are number of features of commercial wheelchairs that do not suit the models which can be simulated within commercial games engines, for example, free-rotating caster wheels are unnecessarily challenging to implement.

Simulation sickness is also a concern for physics models in VR-based simulators. According to the Oculus Rift best practice document [46], acceleration in all forms causes discomfort in users. A kinematic model allows all motion to be carefully controlled, and restricted to comfortable levels where required.

\subsection{Training Tasks}

When wearing a HMD, the trainee is given a first person view of the scene from the perspective of sitting in the virtual wheelchair model - see Figure 2. If the trainee looks down, they will see virtual legs in the position where their real legs would be.

The trainee can access a series of rooms in WheelchairVR, each designed with a different scenario to be completed. We do not attempt to reproduce reality, rather we use abstract tasks that develop the same skills required and used during powered wheelchair navigation. All of the room environments have been built in Unity and additional scenarios can be added as required. The current scenarios consist of:

- Navigating through a simple maze, requiring the user to make several turns, open doors, and maneuver through doorways.

- A goal orientated scenario consisting of navigating through a room filled with floating red and blue balls. The trainee has to collect all of the blue balls by driving in to them. Collisions with the red balls must be avoided.

- Traverse a room that contains a random number of human size capsules that move around the room. This 


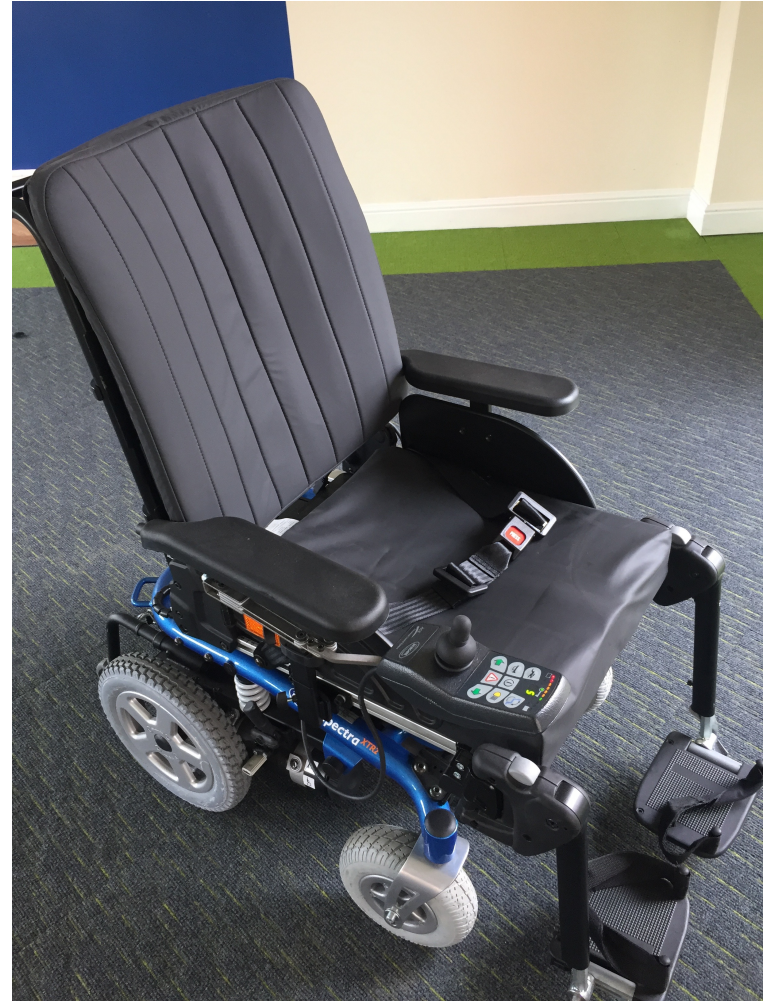

Fig. 1. The Spectra XTR2 Rear Wheel Drive Wheelchair used in the Validation Study

represents driving the wheelchair through a crowded room.

- Navigate through a track made up of kerbstones as quickly as possible, avoiding collisions with a kerbstone.

- A room full of ramps that must be ascended and descended. Time taken is recorded.

Figure 3 provides three example snapshots from these different scenarios.

\section{Validation Study}

The purpose of the validation study was to determine if the VR simulator had any effect on learning the driving skills needed to safely operate a real powered wheelchair.

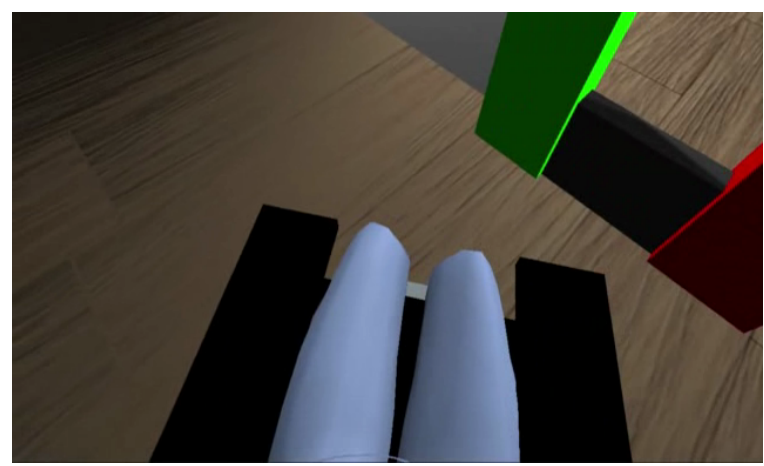

Fig. 2. Wheelchair-VR provides a first person view of the scene from the perspective of sitting in a wheelchair.

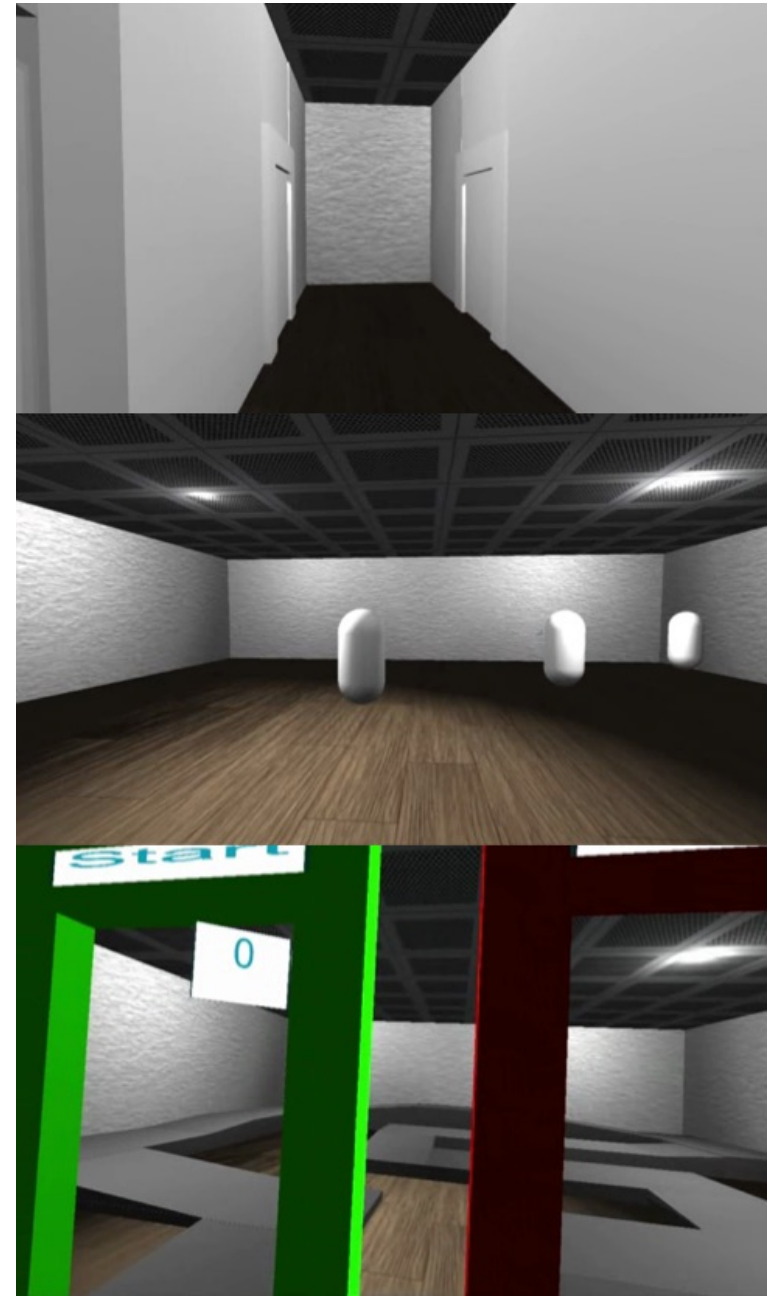

Fig. 3. Snapshots taken from three different rooms in Wheelchair-VR: doorway maze; moving human-size capsules; and ramp challenge.

Typically wheelchair users are only given informational leaflets [15] and then learn to drive through a trial-and error process.

Ethical approval was obtained from the University of Chester Research Ethics Committee, to conduct a validation study using able-bodied volunteers. The design of this study and the results obtained are detailed below.

\subsection{Hardware Components}

Several cost effective head mounted displays have recently become commercially available, including the Oculus Rift (Oculus VR, CA, USA) and HTC Vive (HTC Corp., Taiwan). An advantage of using Unity is that it supports most hardware options, including HMDs and desktop platforms. At the time of the validation study the only HMD available to us was the Oculus Rift DK2 prototype. This provided a low persistence OLED display (which helped minimise motion blur and judder) with 960 by 1080 pixels per eye, and up to $75 \mathrm{~Hz}$ refresh rate. It also offered a $100^{\circ}$ field of view and integrated near infrared positional tracking. Figure 4 shows Wheelchair-VR in use with the trainee wearing the Oculus Rift DK2.

A standard 'gaming' PC or laptop specification is needed to run Wheelchair-VR. In the validation study the PC was 


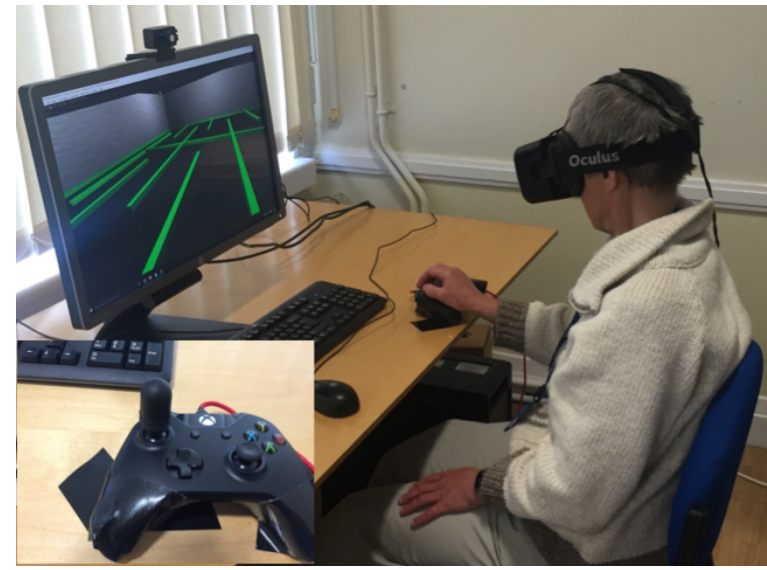

Fig. 4. The Wheelchair-VR Hardware components consist of the PC, an Oculus Rift DK2 HMD, and an XBox controller. Insert shows the adapted joystick on the XBox controller.

a HP Z440 running Windows 10, with Intel Xeon $3.5 \mathrm{GHz}$ CPU, 16 GB RAM and a NVidia Quadro K4200 graphics card.

\subsection{Experimental Design}

Thirty three able-bodied volunteers participated in the validation study, which was the maximum number we were able to recruit on the day. Seven were female and twenty six were male. Their age range was from 20 to over 60 with the majority (19) being under 29. All were beginners in the use of a powered wheelchair and had not driven one before. They were all screened at the beginning of the study to ensure that they could demonstrate a good reaction time. The freely available Reaction Test Pro (Freedom Apps) iPhone App was used for this purpose and all participants achieved under 0.6 seconds, with an average of $0.36 \pm$ 0.08 seconds, which was well within the acceptable range recommended by our collaborators at the local Wheelchair Centre. The participants were randomly divided into three groups of eleven: a Control group who would receive no training; a 'HMD' group who would receive training using the immersive Wheelchair-VR application; and a 'Desktop' group who also used Wheelchair-VR but just with a desktop monitor and not the HMD (see Table 1). The latter replicated the setup used in some of the earlier studies reported in Section 2. Both the HMD and Desktop groups used the gamepad to control the movements of the virtual chair. The HMD group could freely look around the environment by just looking in any direction. The Desktop group were restricted to looking straight ahead - but always had a good view of the obstacles they were negotiating. Note that this initial validation study used all available volunteers and designed to investigate whether there is any indication of a difference between these three groups.

A Spectra XTR2 rear wheel drive wheelchair was used during the study - see Figure 1. To assess the baseline skill of the participants they were all required to drive the Spectra through an obstacle course with the time taken to complete each obstacle recorded. The obstacle course - see Figure 5 consisted of four stages:
1) To manouevre the wheelchair through an open door and enter the room where the obstacle course was laid out.

2) To drive the chair around a circular path, pausing to reach out and flick a wall mounted light switch.

3) To complete a slalom course through six cones, placed $1 \mathrm{~m}$ apart.

4) To reverse park the wheelchair into a tight area marked out by cones.

A time penalty of one second was added for any cone that was hit by the wheelchair. Note that the wheelchair controller was mounted on the left or right arm of the wheelchair depending on the handedness of the participant.

Following the initial attempt at the obstacle course, the HMD and Desktop groups received training using Wheelchair-VR. They were required to complete four of the different room scenarios described in Section 3.3 using a virtual chair configured for rear wheel drive. The XBox controller was placed in the appropriate position for the leftor right-handed participants. Participants were allowed to complete the scenarios in their own time but nobody needed longer than 10 minutes. The HMD group also completed a Simulator Sickness Questionnaire (SSQ) so that any occurrence of cybersickness was recorded [24]. These participants were told to stop using Wheelchair-VR if they started to feel ill. The participants in the control group were not given any training. Instead they were given ten minutes to read a guide to using an electric wheelchair safely [15]. None of the groups were allowed to have extra time in the Spectra between the two attempts of the obstacle course.

All participants then completed the obstacle course in the Spectra a second time, with the time taken and number of cones hit again being recorded.

\subsection{Results}

A one-way analysis of variance (ANOVA) was carried out on the overall timings from the first run of the obstacle course to confirm that there were no significant differences between the three groups (group $p=0.285$ ). Probability plots also confirmed that the assumption that data values follow a normal distribution is valid.

To quantify any driving improvement achieved by an individual participant (less time taken and less obstacles hit), we define an improvement parameter as the difference between each of their two registered times, per stage and overall, divided by the maximum of these two times. This normalized parameter can be expressed as a percentage, and the sign denotes whether the participant got better or worse between the first and second attempt of the obstacle course.

The overall task completion times for the obstacle course were between one and three minutes, and were very depen-

TABLE 1

Group-per-session allocation

\begin{tabular}{l|l|l|l}
\hline & HMD & Desktop & Control \\
\hline Obstacle Course (control) & $\checkmark$ & $\checkmark$ & $\checkmark$ \\
\hline Training Session VR & $\checkmark$ & & \\
\hline Training Session Desktop & & $\checkmark$ & \\
\hline Obstacle Course & $\checkmark$ & $\checkmark$ & $\checkmark$ \\
\hline
\end{tabular}




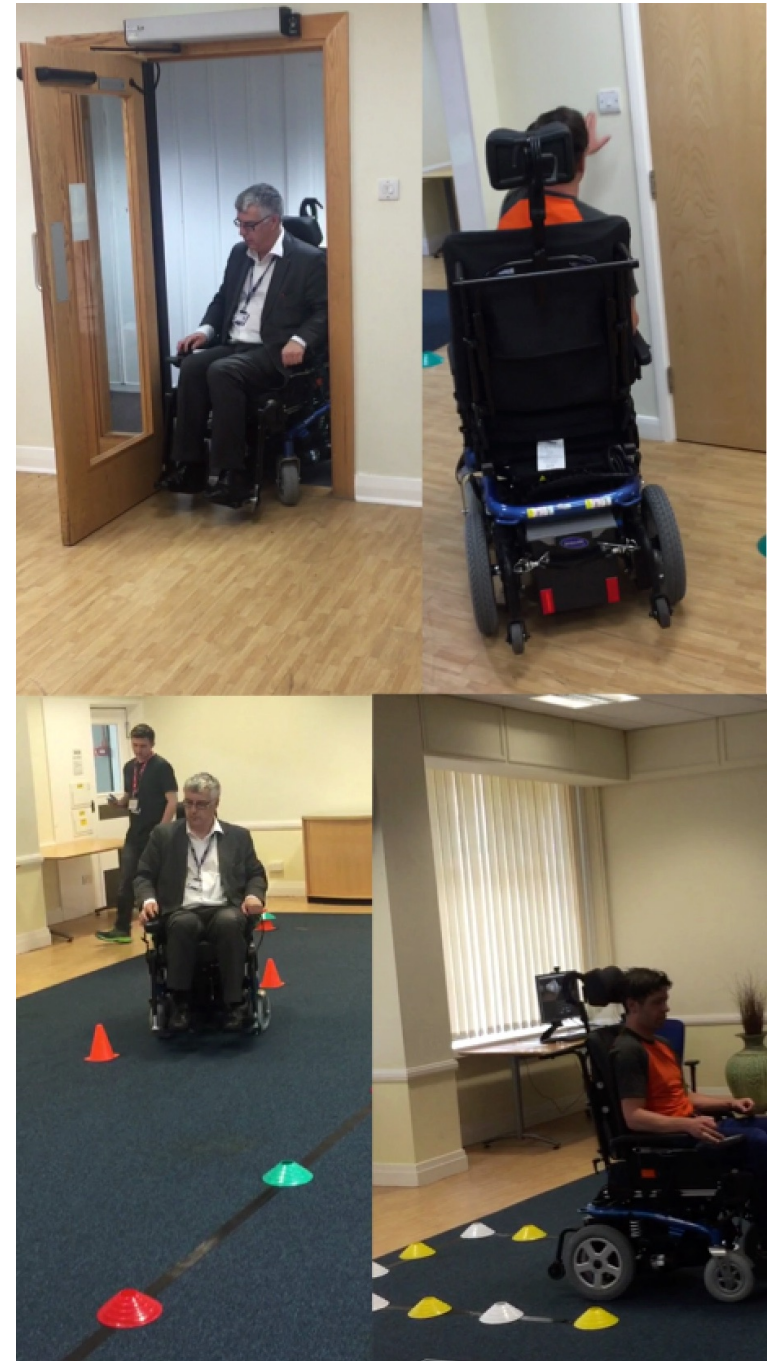

Fig. 5. The four components of the timed obstacle course. Top left: go through doorway. Top right: traverse a circle and reach out to press a light switch. Bottom left: slalom. Bottom right: reverse parking.

dent on the competency of each individual. Therefore the percentage of improvement for each participant is used in the following analysis rather than the absolute completion times. The box plots in Figure 6 summarizes this improvement measure for the three groups over the complete obstacle course, and there is a clear indication that the HMD group is doing better. This is confirmed by examining the mean improvement within each group for all participants. For the Control and Desktop groups this is similar at $16 \%$ and $14 \%$ respectively. A larger mean improvement of $27 \%$ is obtained for the HMD group. A one-way ANOVA was then carried out to determine at what level this difference is significant. This included a contrast analysis for the HMD group verses the other two groups both separately and combined. One-way ANOVAs were also calculated for the four individual stages of the obstacle course. The power values for the ANOVAs (based on a difference in improvement of $10 \%$ with a two-sided $5 \%$ significance level) were: whole obstacle course, 37\%; navigating through narrow door, $10 \%$; circular path with light switch, $58 \%$; slalom $42 \%$; and reverse parking, $27 \%$. The power values are fairly low, which is to be expected with such small sample sizes. Nevertheless significant differences could be detected.

The ANOVA for the improvement across the complete obstacle course produced a $p$-value of 0.083 for the comparison between the three groups. The contrast analysis produced two significant results at the 5\% level: HMD vs Desktop, $p=0.034$, and HMD vs combined Desktop and Control groups, $p=0.030$.

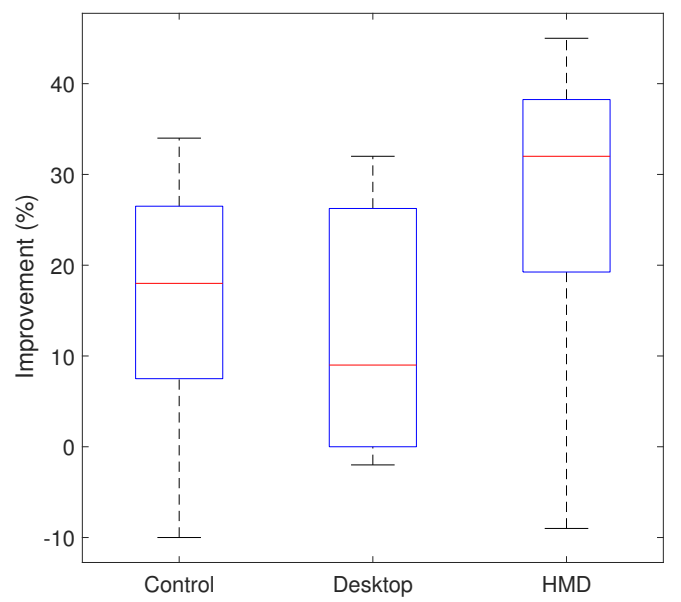

Fig. 6. The improvement across the whole obstacle course. The median is indicated by the horizontal line.

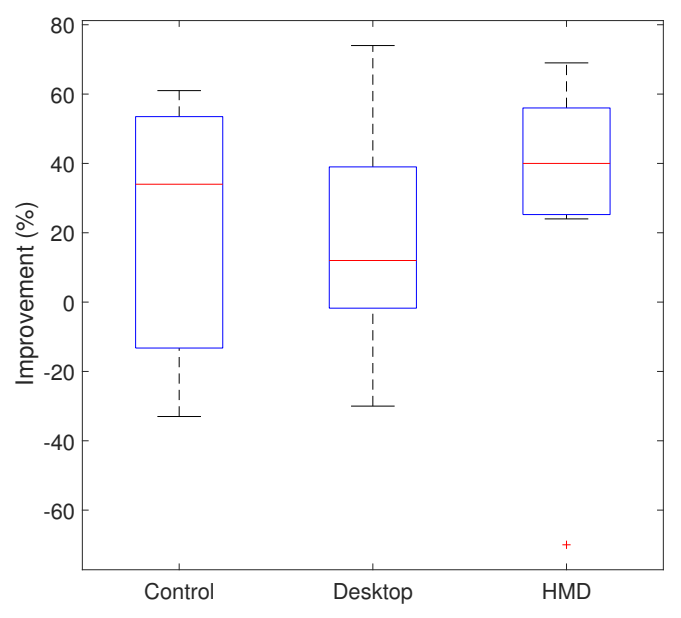

Fig. 7. The improvement for navigating through the narrow door. Outlier with negative improvement is marked with a cross.

A closer analysis of the four separate stages of the obstacle course identifies which were more challenging and where particular performance gains were made. At the first stage, navigating through a narrow door, each group shows a similar improvement between runs and the group $p$-value from the ANOVA is 0.496 . The performances of participants varied greatly (see Figure 7), some of them achieving excellent improvement in the second run $(60-70 \%)$, whereas others had more difficulty navigating through the door at their second attempt and one participant from the HMD 
group got stuck in the door frame $(-70 \%)$. Hence the standard deviation was quite large for this task. If the worst performing participant is treated as an outlier and removed from the ANOVA, then the group p-value becomes 0.084, and the contrast analysis gives two results significant at the $5 \%$ level: HMD vs Desktop, $p=0.031$, HMD vs combined Desktop and Control groups, $p=0.034$.

The second stage, circular path with light switch, is summarized with box plots in Figure 8. The HMD group mean improved by $20 \%$ whereas the Control and Desktop groups both had a $9 \%$ improvement. The group $p$-value from the ANOVA is 0.032 and results from the contrast analysis give: HMD vs Control, $p=0.02$; HMD vs Desktop, $p=0.024$, HMD vs combined Desktop and Control groups, $p=0.009$, all of which are significant at well below the $5 \%$ level.

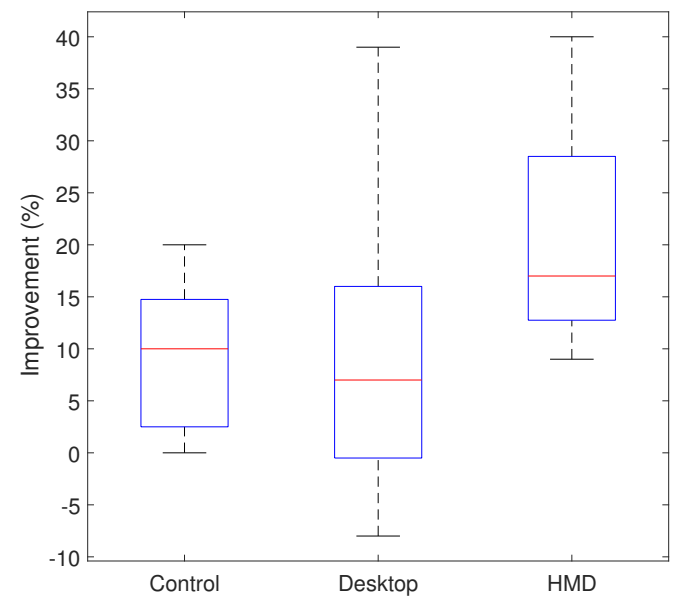

Fig. 8. The improvement for the circular path with light switch

At the third stage, the slalom, the participants in the HMD group again show more improvement than the other two groups (a $22 \%$ mean improvement in comparison with $12-13 \%$ ) - see also box plots in Figure 9. The group $p$ value from the ANOVA is 0.127 . Results from the contrast analysis gives one significant result at the $5 \%$ level: HMD vs combined Desktop and Control groups, $p=0.046$. There are two potential outliers, one in the Desktop group and one in the HMD group, that had very large improvements for this task. If they are removed from their respective groups then the $p$-value from the ANOVA becomes 0.061, and from the contrast analysis: HMD vs Desktop, $p=0.025$; HMD vs combined Desktop and Control groups, $p=0.022$. This indicates that the HMD group's improvement compared to the other groups is significant at below the 5\% level.

For the final reverse parking stage (see box plots in Figure 10) the HMD group show a $29 \%$ mean improvement compared with $10 \%$ and $12 \%$ for the other two groups. The group $p$-value from the ANOVA is 0.026 and significant at the $5 \%$ level. The contrast analysis gave: HMD vs Control, $p=0.027$; HMD vs Desktop, $p=0.013$, HMD vs combined Desktop and Control groups, $p=0.008$. All indicate the the HMD group performed significantly better.

Finally, with the same three outliers referred to above

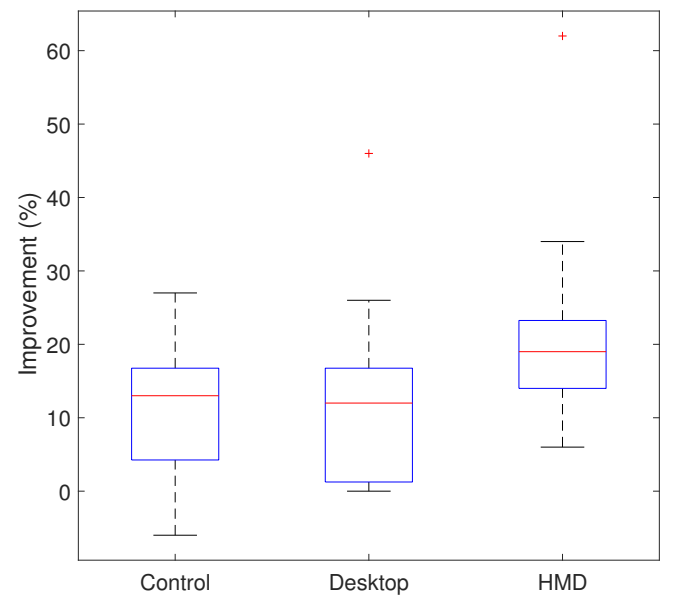

Fig. 9. The improvement for the slalom. Outliers are marked with a cross.

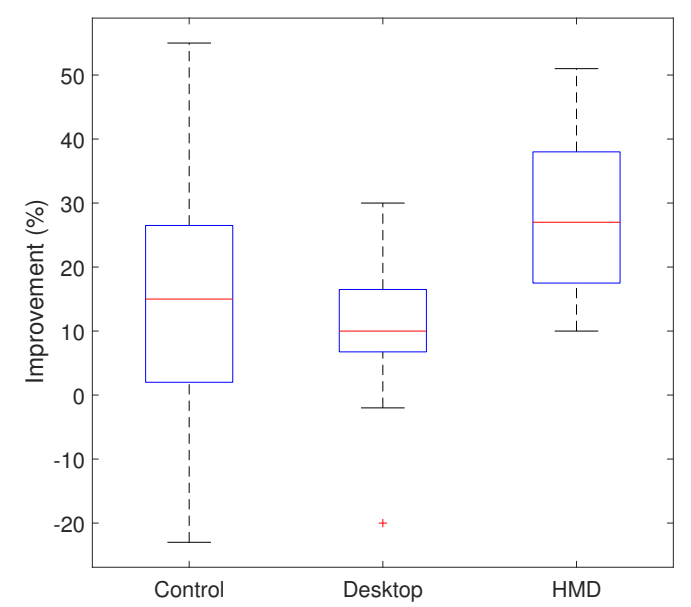

Fig. 10. The improvement for reverse parking. Outlier with negative improvement is marked with a cross.

removed from the ANOVA, then the group $p$-value for the improvement across the complete obstacle course becomes 0.010 .

\subsubsection{Simulator Sickness Questionnaire}

The HMD group completed the SSQ questionnaire devised by Kennedy et al. [24] before and after their training session. We calculated the scores for the three symptom clusters: Nausea (N), Oculomotor (O) and Disorientation (D), along with the Total Severity (TS). The results are summarized in Table 2 for the pre-evaluation symptoms, Table 3 for the post-evaluation symptoms, with Table 4 indicating the score ranges, for each symptom level (none, slight, moderate, severe).

On average the participants reported slight levels of nausea, oculomotor issues and disorientation, with only one participant reporting moderate levels, in all three symptom clusters, after the evaluation. All 11 participants reported a slight increase in discomfort in at least one of the symptoms. None of the participants reported a severe effect. Burping 
was the only symptom not felt by anyone, all other 15 symptoms in the SSQ were increased in at least one participant post-evaluation.

General discomfort was the most common symptom with eight participants reporting a slight or moderate increase following the training session. Six participants also reported a slight or moderate feeling of nausea whilst they had no nausea before the session. Sweating and stomach awareness were also commonly reported following the training session. Only one participant reported vertigo or salivation increasing post-test, and two participants reported a slight headache.

TABLE 2

SSQ - Pre-evaluation Findings

\begin{tabular}{l|c|c|c|c}
\hline & Nausea & Oculomotor & Disorientation & TS \\
\hline Mean & 3.47 & 2.76 & 2.53 & 3.40 \\
\hline SD & 6.43 & 7.01 & 5.63 & 6.78 \\
\hline Min & 0.00 & 0.00 & 0.00 & 0.00 \\
\hline Max & 19.08 & 22.74 & 13.92 & 22.44 \\
\hline
\end{tabular}

TABLE 3

SSQ - Post-evaluation Findings

\begin{tabular}{l|c|c|c|c}
\hline & Nausea & Oculomotor & Disorientation & TS \\
\hline Mean & 32.96 & 22.74 & 41.76 & 35.36 \\
\hline SD & 23.12 & 18.57 & 40.34 & 26.56 \\
\hline Min & 9.54 & 0.00 & 0.00 & 3.74 \\
\hline Max & 76.32 & 68.22 & 139.20 & 100.98 \\
\hline
\end{tabular}

TABLE 4

SSQ - Reference Scores

\begin{tabular}{l|c|c|c|c}
\hline & Nausea & Oculomotor & Disorientation & TS \\
\hline none & 0 & 0 & 0 & 0 \\
\hline slight & 66.8 & 53.1 & 97.4 & 78.5 \\
\hline moderate & 133.6 & 106.1 & 194.9 & 157.1 \\
\hline severe & 200.3 & 159.2 & 292.3 & 235.6 \\
\hline
\end{tabular}

\section{Discussion}

Our initial prediction was that most participants in the validation study would likely improve between the first and second run of the obstacle course, just from the experience of having completed the course once before. This was indeed the case. However, the overall improvement with the HMD group was far better than both the Control and Desktop groups, and showing significance at the $5 \%$ level for some of the particular manoeuvres. The improvement is particularly striking given that the participants only had one short training session. Overall, the improvement between the Control and Desktop groups is comparable. This can be attributed to the participants getting used to wheelchair navigation, in general terms, from subsequent uses. We observe an improvement of about $10 \%-15 \%$ in most cases. However, improvement for the HMD group was observed to be twice, and in cases three times as much (e.g, reverse parking task had $29 \%$ mean improvement). We attribute that, primarily, to the detachment a VR HMD offers from the wearer's surroundings, therefore increasing their focus and training benefits from completing the task at hand, in VR. Compared to the Desktop and Control groups, users do not get distracted from their surroundings. Our claim is supported by the findings of Thalmann et al. [40], who highlight that with a HMD, the sense of presence becomes stronger, due to the link with the real world being diminished.

On a secondary level, the results also indicate that using the training software with a desktop monitor offers no training benefit from just a single use. Over an extended period of time with multiple training sessions, desktop training can be effective, as was demonstrated by Inman et al. [22]. In fact, Buttussi and Chittaro [8] found that desktop training can have similar benefits (increased knowledge and self-efficacy), regardless of the used display. Nonetheless, their evaluation spans a period of over two weeks, whereas for our study the investigations took place back-to-back within a few hours. Our approach fits well with how posture and mobility professionals at the Wheelchair Centres, often restricted in time and resources, would have to train prospective wheelchair users.

Looking into each of the four tasks that made up the obstacle course, the improvement did vary. For both the traversal of a circular path with light switch task, and the reverse parking task, it is clear that the HMD group improved significantly at the $5 \%$ level compared with the other groups. For the slalom task, the improvement is not as significant, yet still observable. A possible explanation is that the spatial awareness and navigation understanding of the HMD group was helped more by being immersed in the VR environment. Compared to the Desktop version, the HMD with its head-tracking offers better interaction fidelity [8], which in turn seems to have a positive effect in learning to manoevre in VR, and transferring said learning to the physical world. On the other hand, compared to the physical wheelchair it is less intimidating to use, more engaging and inspires confidence. The latter was especially highlighted by our post-experiment participants' comments on the HMD version, such as: "It built confidence for use of the real chair.", "Getting used to wheelchair joystick without bumping into things!" and "Gave confidence. These [tasks] would take longer in a life practice situation."

There is less evidence that any group did significantly better in learning how to manouevre through a physical doorway, which seemed to be the most challenging task, despite the fact the physical doorway was designed for wheelchair access and could be opened automatically. We attribute this to VR training systems being predominately non-contact [38]. In our implementation negotiating doorways in VR is considerably less difficult and hazardous than in the real world, where users need to be extra careful not to crash into walls and hurt their fingers, knees, etc.. Consequently, we can assume that to simulate such tasks realistically, such as negotiating doorways and corridors, we would need some form of force feedback for when we hit the doorway. Moreover, as discussed by Stott and Sanders [38], training for such fine adjustments in navigation of the physical wheelchair can not be simulated in VR without mechanisms that convincingly replicate the wheelchair's physical properties (e.g., weight, rigidity, etc.) and movement char- 
acteristics, such as breaking smoothness, rotational acceleration, and friction when sliding around physical objects. Harrison et al. [18] built a wheelchair platform that applied force directly to the rims of the wheels and so demonstrate that providing the users with this sort of response would be possible. Interestingly, besides the outlier, for the remaining members of the group we do observe a significant improvement for this task, which in turn indicates that further investigation of similar scenarios is needed.

Regarding cybersickness, the results from the SSQ indicate that the mismatch between physical and virtual motion in Wheelchair-VR for the HMD Group is a problem, even with a relatively short training session. The user remains seated but stationary in the real world, whilst apparently moving around in the virtual wheelchair. This will cause neural conflict and is a well known source of cybersickness. We have recently tried running Wheelchair-VR with the consumer version of the Oculus Rift. Initial feedback indicates that the amount of cybersickness is reduced - most likely due to the better resolution, higher refresh rate, and tracking improvements of the newer device. However, some degree of cybersickness persists. We also noted that the participants who only reported minimal effects of cybersickness tended to look downwards as they drove the wheelchair, where they could see their virtual legs or the arms of the chair. It is possible that having a point of reference that was moving with the chair helped to reduce cybersickness. A recent study on including an image of a virtual nose as a point of reference in the centre of the field of view of an Oculus Rift supports this theory [44].

\section{Conclusions ANd Future Work}

We have created and demonstrated the utility of a costeffective virtual environment for powered wheelchair training, built from commercial off-the-shelf components. Our findings support our hypothesis that a serious game (virtual environment) utilising the Oculus Rift HMD can provide a safe environment in which a new user of a powered wheelchair can quickly learn driving skills. Our results indicate that: a) the learning done in the HMD-based VR environment was transferred to the physical world; and b) there is a benefit in using a VR-HMD simulator for training wheelchair users, in terms of navigation performance improvement. Coupling that with the safety that users felt and overall confidence the HMD version provided, there are clear benefits in using HMD-based VR for training wheelchair users.

One of the aspect we want to improve in future incarnations of our system is to reduce the occurrence of cybersickness. One technique that has helped in that regard, in particular with an Oculus Rift, is to change a physically stationary persons field of view in response to visually perceived motion [14]. This approach will be investigated for our application. Once the cybersickness problem has been alleviated then we will perform a second validation study with volunteers who are actual wheelchair users. Our Wheelchair Centre collaborators will help to facilitate this.

As well as addressing cybersickness, force-feedback, allowing the user to feel the virtual environment through the wheelchair, is another area needing further research that we identified in Section 2. We expect this to enhance the overall sense of immersion and make training tasks such as negotiating narrow doorways and corridors, and slalom, more effective. We have begun investigating an approach that uses a force feedback device as the controller for our simulated wheelchair. This will only provide force feedback to the users hand but even this limited haptics interface may be beneficial. Scenarios with uneven floor surfaces and collisions with objects have been implemented but a study to investigate any training benefits will be future work.

\section{ACKNOWLEDGMENT}

The authors wish to thank Invacare UK for the loan of the Spectra wheelchair. We also acknowledge the help and assistance given by Liverpool Wheelchair Service, Wirral Community NHS Trust Wheelchair Service, Cardiff and Vale UHB Posture Mobility Centre, and Betsi Cadwaladr UHB Posture and Mobility Service. Finally we thank Dr Shirley John for her invaluable assistance with the statistical analysis.

\section{REFERENCES}

[1] S. V. Adamovich, G. G. Fluet, E. Tunik, and A. S. Merians. Sensorimotor training in virtual reality: a review. NeuroRehabilitation, 25(1):29-44, 2009.

[2] P. B. Andreatta, E. Maslowski, S. Petty, W. Shim, M. Marsh, T. Hall, S. Stern, and J. Frankel. Virtual reality triage training provides a viable solution for disaster-preparedness. Academic emergency medicine, 17(8):870-876, 2010.

[3] J. N. Bailenson, N. Yee, J. Blascovich, A. C. Beall, N. Lundblad, and M. Jin. The use of immersive virtual reality in the learning sciences: Digital transformations of teachers, students, and social context. The Journal of the Learning Sciences, 17(1):102-141, 2008.

[4] B. Bideau, F. Multon, R. Kulpa, L. Fradet, and B. Arnaldi. Virtual reality applied to sports: Do handball goalkeepers react realistically to simulated synthetic opponents? In Proceedings of the 2004 ACM SIGGRAPH International Conference on Virtual Reality Continuum and Its Applications in Industry, VRCAI '04, pages 210216, New York, NY, USA, 2004. ACM.

[5] J. P. Bliss, P. D. Tidwell, and M. A. Guest. The effectiveness of virtual reality for administering spatial navigation training to firefighters. Presence: Teleoperators and Virtual Environments, 6(1):73-86, 1997.

[6] C. J. Bohil, B. Alicea, and F. A. Biocca. Virtual reality in neuroscience research and therapy. Nature reviews neuroscience, 12(12):752-762, 2011.

[7] K. Brütsch, T. Schuler, A. Koenig, L. Zimmerli, S. Mérillat, L. Lünenburger, R. Riener, L. Jäncke, and A. Meyer-Heim. Influence of virtual reality soccer game on walking performance in robotic assisted gait training for children. Journal of neuroengineering and rehabilitation, 7(1):15, 2010.

[8] F. Buttussi and L. Chittaro. Effects of different types of virtual reality display on presence and learning in a safety training scenario. IEEE Transactions on Visualization and Computer Graphics, 2017.

[9] R. Cooper, D. Spaeth, D. Jones, M. Boninger, S. Fitzgerald and S. Guo. Comparison of virtual and real electric powered wheelchair driving using a position sensing joystick and an isometric joystick. Medical Engineering E Physics, 24(10):703-708, 2002.

[10] J. Dascal, M. Reid, W. W. IsHak, B. Spiegel, J. Recacho, B. Rosen, and I. Danovitch. Virtual reality and medical inpatients: A systematic review of randomized, controlled trials. Innovations in Clinical Neuroscience, 14(1-2):14, 2017.

[11] E. de Bruin, D. Schoene, G. Pichierri, and S. Smith. Use of virtual reality technique for the training of motor control in the elderly. Zeitschrift für Gerontologie und Geriatrie, 43(4):229-234, 2010.

[12] M. Desbonnet, S. Cox, and A. Rahman. Development and evaluation of a virtual reality based training system for disabled children. In Proceedings of the European Conference on Disability, Virtual Reality and Associated Technologies, volume 11, pages 177-182, 1998. 
[13] B. Faria, L. Reis, and N. Lau. A Survey on Intelligent Wheelchair Prototypes and Simulators. In New Perspectives in Information Systems and Technologies, volume 1, pages 545-557. Springer, 2014.

[14] A. S. Fernandes and S. K. Feiner. Combating vr sickness through subtle dynamic field-of-view modification. In 2016 IEEE Symposium on 3D User Interfaces (3DUI), pages 201-210. IEEE, 2016.

[15] O. C. for Enablement. A guide to using an electric wheelchair safely. Brochure, 2011.

[16] M. Grant, C. Harrison, and B. Conway. Wheelchair simulation. In Proceedings of the Cambridge Workshop Series on Universal Access and Assistive Technology, 2004.

[17] A. Harrison, G. Derwent, A. Enticknap, F. Rose, and E. Attree. The role of virtual reality technology in the assessment and training of inexperienced powered wheelchair users. Disability $\mathcal{E}$ Rehabilitation, 24(11):599-606, 2002.

[18] C. Harrison, M. Grant, and B. Conway. Haptic interfaces for wheelchair navigation in the built environment. Presence: Teleoperators and Virtual Environments, 13(5):520-534, 2004.

[19] C. Harrison, P. Grant, and B. Conway. Enhancement of a virtual reality wheelchair simulator to include qualitative and quantitative

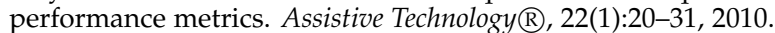

[20] C. J. Headleand, T. Day, S. R. Pop, P. D. Ritsos, and N. W. John. A cost-effective virtual environment for simulating and training powered wheelchairs manoeuvres. Proceedings of NextMed/MMVR22, 2016.

[21] D. Inman, K. Loge, and J. Leavens. VR Education and Rehabilitation. Communications of the ACM, 40(8):53-58, 1997.

[22] D. P. Inman, K. Loge, A. Cram, and M. Peterson. Learning to drive a wheelchair in virtual reality. Journal of Special Education Technology, 26(3):21-34, 2011.

[23] N. W. John, N. I. Phillips, L. ap Cenydd, D. Coope, N. CarletonBland, I. Kamaly-Asl, and W. P. Gray. A tablet-based virtual environment for neurosurgery training. Presence: Teleoperators and Virtual Environments, 24(2):155-162, 2015.

[24] R. S. Kennedy, N. E. Lane, K. S. Berbaum, and M. G. Lilienthal. Simulator sickness questionnaire: An enhanced method for quantifying simulator sickness. The international journal of aviation psychology, 3(3):203-220, 1993.

[25] L. Kiss, B. P. Hámornik, M. Köles, P. Baranyi, P. Galambos, and G. Persa. Training of business skills in virtual reality. In Cognitive Infocommunications (CogInfoCom), 2015 6th IEEE International Conference on, pages 215-216. IEEE, 2015.

[26] J. Kozak, P. Hancock, E. Arthur, and S. Chrysler. Transfer of training from virtual reality. Ergonomics, 36(7):777-784, 1993.

[27] J. Li, L. Khoo, and S. Tor. Desktop virtual reality for maintenance training: an object oriented prototype system (v-realism). Computers in Industry, 52(2):109 - 125, 2003.

[28] M. Linden, C. Whyatt, C. Craig, and C. Kerr. Efficacy of a powered wheelchair simulator for school aged children: a randomized controlled trial. Rehabilitation psychology, 58(4):405, 2013.

[29] R. B. Loftin and P. Kenney. Training the hubble space telescope flight team. IEEE Computer Graphics and Applications, 15(5):31-37, Sep 1995.

[30] V. Luboz, Y. Zhang, S. Johnson, Y. Song, C. Kilkenny, C. Hunt, H. Woolnough, S. Guediri, J. Zhai, T. Odetoyinbo, et al. Imagine seldinger: First simulator for seldinger technique and angiography training. Computer methods and programs in biomedicine, 111(2):419434, 2013.

[31] H. P. Mahajan, B. E. Dicianno, R. A. Cooper, and D. Ding. Assessment of wheelchair driving performance in a virtual reality-based simulator. The journal of spinal cord medicine, 36(4):322-332, 2013.

[32] K. I. Molina, N. A. Ricci, S. A. de Moraes, and M. R. Perracini. Virtual reality using games for improving physical functioning in older adults: a systematic review. Journal of NeuroEngineering and Rehabilitation, 11(1):156, 2014

[33] T. Mujber, T. Szecsi, and M. Hashmi. Virtual reality applications in manufacturing process simulation. Journal of Materials Processing Technology, 155156:1834 - 1838, 2004. Proceedings of the International Conference on Advances in Materials and Processing Technologies: Part 2.

[34] T. Pithon, T. Weiss, S. Richir, and E. Klinger. Wheelchair simulators: A review. Technology and Disability, 21(1):1-10, 2009.

[35] N. Rodriguez. Development of a wheelchair simulator for children with multiple disabilities. In Proceedings of the 3rd IEEE VR International Workshop on Virtual and Augmented Assistive Technology (VAAT), pages 19-21. IEEE, 2015.
[36] N. E. Seymour, A. G. Gallagher, S. A. Roman, M. K. Obrien, V. K. Bansal, D. K. Andersen, and R. M. Satava. Virtual reality training improves operating room performance: results of a randomized, double-blinded study. Annals of surgery, 236(4):458-464, 2002.

[37] M. Slater and M. V. Sanchez-Vives. Enhancing our lives with immersive virtual reality. Frontiers in Robotics and AI, 3:74, 2016.

[38] I. Stott and D. Sanders. The use of virtual reality to train powered wheelchair users and test new wheelchair systems. International Journal of Rehabilitation Research, 23(4):321-326, 2000.

[39] D. Stredney, W. Carlson, L. Swan, J. Edward, and B. Blostein. The Determination of Environmental Accessibility and ADA Compliance Through Virtual Wheelchair Simulation. PresenceTeleoperators and Virtual Environments, 4(3):297-305, 1995.

[40] D. Thalmann, J. Lee, and N. M. Thalmann. An evaluation of spatial presence, social presence, and interactions with various $3 \mathrm{~d}$ displays. In Proceedings of the 29th International Conference on Computer Animation and Social Agents, pages 197-204. ACM, 2016.

[41] J. Tichon and R. Burgess-Limerick. A review of virtual reality as a medium for safety related training in mining. Journal of Health $\mathcal{E}$ Safety Research \& Practice, 3(1):33-40, 2011.

[42] E. van Wyk and R. de Villiers. Virtual reality training applications for the mining industry. In Proceedings of the 6th International Conference on Computer Graphics, Virtual Reality, Visualisation and Interaction in Africa, AFRIGRAPH '09, pages 53-63, New York, NY, USA, 2009. ACM.

[43] P.-F. Villard, F. P. Vidal, L. ap Cenydd, R. Holbrey, S. Pisharody, S. Johnson, A. Bulpitt, N. W. John, F. Bello, and D. Gould. Interventional radiology virtual simulator for liver biopsy. International journal of computer assisted radiology and surgery, 9(2):255-267, 2014

[44] D. Whittinghill, B. Ziegler, T. Case, and B. Moore. Nasum virtualis: A simple technique for reducing simulator sickness. In Games Developers Conference (GDC), 2015.

[45] P. N. Wilson, N. Foreman, and M. Tlauka. Transfer of spatial information from a virtual to a real environment. Human Factors, 39(4):526-531, 1997.

[46] R. Yao, T. Heath, A. Davies, T. Forsyth, N. Mitchell, and P. Hoberman. Oculus vr best practices guide. Oculus VR, 2014.

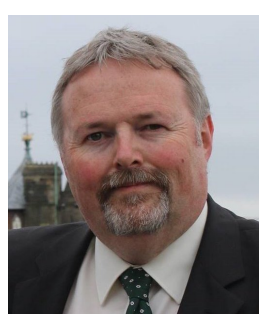

Nigel W. John has been a Senior Research Professor within the Department of Computer Science of the University of Chester since July 2015. Previously, he held a chair in computing at the School of Computer Science at Bangor University, where he was a director of the pan-Wales Research Institute of Visual Computing (RIVIC). In 2006 he was awarded the 12th annual Satava Award to acknowledge his accomplishments in the field of computer graphics and medical visualization. He is a Fellow of the Eurographics Association and the Learned Society of Wales.

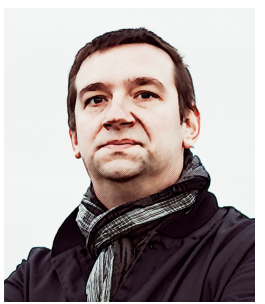

Serban R. Pop is a Senior Lecturer in the Department of Computer Science, UNiversity of Chester. He was formerly a Lecturer at Oxford Brookes University, a Research Officer in the Advanced Medical Imaging and Visualization Unit of the School of Computer Science, Bangor University and a Research Fellow at the School of Mathematical Sciences, Centre for Mathematical Medicine, University of Nottingham. 


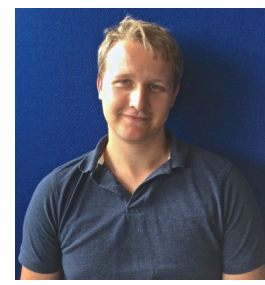

Thomas W. Day completed his B.Sc. in Computer Science at Bangor University, UK in 2015. $\mathrm{He}$ is currently studying for a PhD in the use of Virtual and Mixed Reality in medicine at the University of Chester. His research interests include Mixed and Virtual Reality.

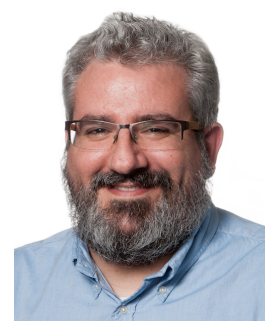

Panagiotis D. Ritsos received the $\mathrm{PhD}$ degree from the University of Essex, UK in 2006. He is currently a Lecturer in the School of Computer Science, Bangor University, UK. His research interests include Mixed and Virtual Reality, Data Visualization and Wearable Computing. $\mathrm{He}$ is a member of the IEEE.

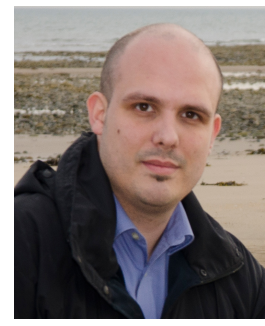

Christopher J. Headleand completed his MSc and $\mathrm{PhD}$ as part of the graphics group at Bangor University. He is currently lecturer at the School of Computer Science, University of Lincoln, UK, and a member of the IntLab research group. His academic interests include virtual worlds, procedural content, ethics, and human-computer interaction. Chris is also the managing director of Picselica, a company specializing in Virtual Reality applications. 